\title{
Four-Dimensional Pseudo-Riemannian Generalized Symmetric Spaces Which are Algebraic Ricci Solitons
}

\author{
W. Batat and K. Onda
}

\begin{abstract}
We classify, up to isometry, non-symmetric simply-connected four-dimensional pseudo-Riemannian generalized symmetric spaces which are algebraic Ricci solitons. It turns out that those of Cerny-Kowalski's types A, C and D are algebraic Ricci solitons, whereas those of type B are not. Thus, we give new examples of algebraic Ricci solitons.
\end{abstract}

Mathematics Subject Classification (2000). 53C50, 53C21, 53C25.

Keywords. Pseudo-Riemannian metrics, generalized symmetric spaces, algebraic Ricci solitons.

\section{Introduction}

The concept of algebraic Ricci soliton was introduced by Lauret in the Riemannian case $([9])$. The definition extends to the pseudo-Riemannian case as follows.

Definition 1.1. Let $(G, g)$ be a simply-connected Lie group equipped with a left-invariant pseudo-Riemannian metric $g$, and let $\mathfrak{g}$ denote the Lie algebra of $G$. The metric $g$ is called an algebraic Ricci soliton if it satisfies

$$
\text { Ric }=c \mathrm{Id}+\mathrm{D}
$$

W. Batat was supported by funds of JSPS; ENPO; and Ministry of Economy and Competitiveness, Spain, under project MTM2011-22528. K. Onda was partially supported by the JSPS Institutional Program for Young Researcher Overseas Visits Promoting international young researchers in mathematics and mathematical sciences led by OCAMIh. 
where Ric denotes the Ricci operator, $c$ is a real number, and $\mathrm{D} \in \operatorname{Der}(\mathfrak{g})$, that is,

$$
\mathrm{D}[X, Y]=[\mathrm{D} X, Y]+[X, \mathrm{D} Y], \quad \text { for all } \quad X, Y \in \mathfrak{g} .
$$

In particular, an algebraic Ricci soliton on a solvable Lie group (resp. a nilpotent Lie group) is called a solvsoliton (resp. a nilsoliton).

Obviously, Einstein metrics on a Lie group are algebraic Ricci solitons.

A Ricci soliton metric $g$ on a manifold $M$ is a pseudo-Riemannian metric such that there exists a vector field on $X$ satisfying

$$
\varrho_{g}=c g+L_{X} g,
$$

where $L$ denotes the Lie derivative, $\varrho$ is the Ricci tensor and $c$ is a real constant.

The condition (1.3) is equivalent to $g_{t}=(-2 c t+1) \varphi_{s(t)}^{*} g$ being a solution of the Ricci flow

$$
\frac{\partial}{\partial t}\left(g_{t}\right)_{i j}=-2\left(\varrho_{g_{t}}\right)_{i j}
$$

where $\varphi_{s}$ is the family of diffeomorphisms generated by $X$ which one reparametrizes to $s(t)=\frac{1}{c} \ln (-2 c t+1)$.

In [9], Lauret studied the relation between solvsolitons and Ricci solitons on Riemannian manifolds. More precisely, he proved that any left-invariant Riemannian solvsoliton metric is a Ricci soliton. This was extended by the second author to the pseudo-Riemannian case as follows.

Theorem 1.2. ([10]) Let $(G, g)$ be a simply-connected Lie group endowed with a left-invariant pseudo-Riemannian metric $g$. If $g$ is a solvsoliton, then $g$ is a Ricci soliton, that is, $g$ satisfies (1.3), with

$$
X=\left.\frac{d \varphi_{t}}{d t}\right|_{t=0}(p) \quad \text { and } \quad \exp \left(\frac{t}{2} \mathrm{D}\right)=\left.\mathrm{d} \varphi_{t}\right|_{\mathrm{e}}
$$

where $e$ denotes the identity element of $G$.

Note that changing "solvsoliton" to "algebraic Ricci soliton" the theorem above is correct.

On the other hand, if $(M, g)$ is a homogeneous (pseudo-)Riemannian manifold, there exists a group $G$ of isometries acting transitively on it [11]. Such $(M, g)$ can be then identified with $(G / H, g)$, where $H$ is the isotropy group at a fixed point $p$ of $M$. Let $\mathfrak{g}$ denote the Lie algebra of $G$ and fix an $\operatorname{Ad}(H)$-invariant decomposition $\mathfrak{g}=\mathfrak{h} \oplus \mathfrak{m}$, where $\mathfrak{h}$ is the Lie algebra of $H$. The space $\mathfrak{m}$ is naturally identified with $T_{p} M$. In the Riemannian case such a decomposition always exists, since homogeneous Riemannian manifolds are reductive. In the general pseudo-Riemannian, reductivity should generally instead be imposed. Now, for instance, a three-dimensional homogeneous Lorentzian manifold is necessarily reductive. This was proved in [7] and it also follows independently from the classification obtained by Calvaruso in [2]. Furthermore, the existence 
of non-reductive four-dimensional pseudo-Riemannian homogeneous manifolds was proven in [7].

Homogeneous Ricci solitons have been investigated in [8]. A natural generalization of algebraic Ricci solitons on Lie groups to homogeneous spaces is the following [8].

Definition 1.3. Let $(M=G / H, g)$ be a homogeneous Riemannian manifold. Then $g$ is called an algebraic Ricci soliton if

$$
\text { Ric }=c \mathrm{Id}+\operatorname{pr} \circ \mathrm{D}
$$

where Ric denotes the Ricci operator on $\mathfrak{m}$, pr: $\mathfrak{g} \rightarrow \mathfrak{m}$ is the orthogonal projection map, $c$ is a real number, and $\mathrm{D} \in \operatorname{Der}(\mathfrak{g})$.

Note that the above definition can be extended to the pseudo-Riemannian case, changing "homogeneous Riemannian manifold" to "reductive homogeneous pseudo-Riemannian manifold". In [1], we obtained the classification of three-dimensional Lorentzian Lie groups which are algebraic Ricci solitons.

In [4], pseudo-Riemannian four-dimensional generalized symmetric spaces have been classified into four classes, named A, B, C and D, and the (pseudo-) Riemannian metrics can have any signature. All these spaces are reductive homogeneous.

In [3] and [6], the Levi-Civita connection, the curvature tensor and the Ricci tensor of these spaces are computed; proving that type $\mathrm{C}$ is symmetric, that is, the covariant derivative of its curvature tensor vanishes at each point. We will use the results of these computations to study which types of these spaces are algebraic Ricci solitons.

The main result of this paper can be stated (cf. Theorems 3.3, 4.3, 5.3, 6.3) as follows.

Theorem 1.4. Non-symmetric simply-connected four-dimensional pseudo-Riemannian generalized symmetric spaces of type $A, C$ and $D$ in Cerny-Kowalski's classification are algebraic Ricci solitons, whereas those of type $B$ are not.

\section{Preliminaries}

We start by recalling the definition of generalized symmetric space. Let $(M, g)$ be a (pseudo-)Riemannian manifold. A regular s-structure on $M$ is a family of isometries $\left\{s_{p} \mid p \in M\right\}$ of $(M, g)$ such that

- the mapping $M \times M \rightarrow M,(p, q) \mapsto s_{p}(q)$, is smooth,

- $p$ is an isolated fixed point of $s_{p}, \forall p \in M$,

- $s_{p} \circ s_{q}=s_{s_{p}(q)} \circ s_{p}, \forall p, q \in M$.

The map $s_{p}$ is called the symmetry centered at $p$. The order of a regular $s$-structure is the smallest integer $k \geqslant 2$ such that $s_{p}^{k}=\operatorname{id}_{M}$ for all $p \in M$. If such an integer does not exist, we say that the regular $s$-structure has order 
infinity. A generalized symmetric space is a connected, pseudo-Riemannian manifold, carrying at least one regular $s$-structure. In particular, a generalized symmetric space is a pseudo-Riemannian symmetric space if and only if it admits a regular $s$-structure of order 2 . The order of a generalized symmetric space is the minimum of orders of all possible $s$-structures on it. Furthermore, if $(M, g)$ is a generalized symmetric space then it is homogeneous, that is, the full isometry group $I(M)$ of $M$ acts transitively on it, which means that $(M, g)$ can be identified with $(G / H, g)$, where $G \subset I(M)$ is a subgroup of $I(M)$ acting transitively on $M$ and $H$ is the isotropy group at a fixed point $o \in M$.

Generalized symmetric spaces of low dimension have been completely classified. The following theorem recalls the classification of non-symmetric simply-connected four-dimensional pseudo-Riemannian generalized symmetric spaces.

Theorem 2.1. (Cerny and Kowalski [4]) Non-symmetric, simply-connected generalized symmetric spaces $(M, g)$ of dimension 4 are of order either 3 or 4 , or infinity. All these spaces are indecomposable, and belong, up to isometry, to one of the following four types.

- Type A. The underlying homogeneous space is $G / H$, where

$$
G=\left(\begin{array}{lll}
a & b & u \\
c & d & v \\
0 & 0 & 1
\end{array}\right), \quad H=\left(\begin{array}{ccc}
\cos t & -\sin t & 0 \\
\sin t & \cos t & 0 \\
0 & 0 & 1
\end{array}\right),
$$

with $a d-b c=1 .(M, g)$ is the space $\mathbb{R}^{4}(x, y, u, v)$ with the pseudo-Riemannian metric

$$
\begin{aligned}
g= & \lambda\left[\left(1+y^{2}\right) d x^{2}+\left(1+x^{2}\right) d y^{2}-2 x y d x d y\right] /\left(1+x^{2}+y^{2}\right) \\
& \pm\left[\left(-x+\sqrt{1+x^{2}+y^{2}}\right) d u^{2}\right. \\
& \left.+\left(x+\sqrt{1+x^{2}+y^{2}}\right) d v^{2}-2 y^{2} d u d v\right],
\end{aligned}
$$

where $\lambda \neq 0$ is a real constant. The order is $k=3$ and the possible signatures are $(4,0),(2,2)$ and $(0,4)$.

- Type B. The underlying homogeneous space is $G / H$, where

$$
G=\left(\begin{array}{cccc}
e^{-(x+y)} & 0 & 0 & a \\
0 & e^{x} & 0 & b \\
0 & 0 & e^{y} & c \\
0 & 0 & 0 & 1
\end{array}\right), \quad H=\left(\begin{array}{cccc}
1 & 0 & 0 & -w \\
0 & 1 & 0 & -2 w \\
0 & 0 & 1 & 2 w \\
0 & 0 & 0 & 1
\end{array}\right) .
$$

$(M, g)$ is the space $\mathbb{R}^{4}(x, y, u, v)$ with the pseudo-Riemannian metric

$$
g=\lambda\left(d x^{2}+d y^{2}+d x d y\right)+e^{-y}(2 d x+d y) d v+e^{-x}(d x+2 d y) d u
$$

where $\lambda$ is a real constant. The order is $k=3$ and the signature is always $(2,2)$. 
- Type C. The underlying homogeneous space $G / H$ is the matrix group

$$
G=\left(\begin{array}{cccc}
e^{-t} & 0 & 0 & x \\
0 & e^{t} & 0 & y \\
0 & 0 & 1 & z \\
0 & 0 & 0 & 1
\end{array}\right)
$$

$(M, g)$ is the space $\mathbb{R}^{4}(x, y, z, t)$ with the pseudo-Riemannian metric

$$
g= \pm\left(e^{2 t} d x^{2}+e^{-2 t} d y^{2}\right)+d z d t .
$$

The order is $k=3$ and the possible signatures are $(1,3),(3,1)$.

- Type D. The underlying homogeneous space is $G / H$ where

$$
G=\left(\begin{array}{ccc}
a & b & x \\
c & d & y \\
0 & 0 & 1
\end{array}\right), \quad H=\left(\begin{array}{ccc}
e^{t} & 0 & 0 \\
0 & e^{-t} & 0 \\
0 & 0 & 1
\end{array}\right)
$$

with $a d-b c=1$. $(M, g)$ is the space $\mathbb{R}^{4}(x, y, u, v)$ with the pseudo-Riemannian metric

$$
\begin{aligned}
g= & -2 \cosh (2 u) \cos (2 v) d x d y+\lambda\left(d u^{2}-\cosh ^{2}(2 u) d v^{2}\right) \\
& +(\sinh (2 u)-\cosh (2 u) \sin (2 v)) d x^{2} \\
& +(\sinh (2 u)+\cosh (2 u) \sin (2 v)) d y^{2},
\end{aligned}
$$

where $\lambda \neq 0$ is a real constant. The order is infinite and the signature is $(2,2)$.

\section{Spaces of Type A with Neutral Signature}

Let $(M, g)$ be a four-dimensional generalized pseudo-Riemannian symmetric space and denote by $\nabla$ and $R$ the Levi-Civita connection and the Riemann curvature tensor of $M$, respectively. Throughout this paper, we will use the sign convention

$$
R(X, Y)=\nabla_{[X, Y]}-\left[\nabla_{X}, \nabla_{Y}\right]
$$

The Ricci tensor $\varrho$ of $(M, g)$ is defined by

$$
\varrho(X, Y)=\sum_{k=1}^{4} \varepsilon_{k} g\left(R\left(X, e_{k}\right) Y, e_{k}\right),
$$

where $\left\{e_{1}, e_{2}, e_{3}, e_{4}\right\}$ is a pseudo-orthonormal frame field, with $g\left(e_{k}, e_{k}\right)=\varepsilon_{k}=$ \pm 1 . The Ricci operator Ric is then given by

$$
\varrho(X, Y)=g(\operatorname{Ric}(X), Y) .
$$

Now, consider a non-symmetric simply-connected four-dimensional generalized symmetric space $(M=G / H, g)$ of type A and signature $(2,2)$. Then, taking into account the results of [4] and [5], the Lie algebra $\mathfrak{g}$ of the Lie group 
$G$ may be decomposed into the vector space direct sum $\mathfrak{g}=\mathfrak{h} \oplus \mathfrak{m}$ where $\mathfrak{h}$ denotes the Lie algebra of $H$ and $\mathfrak{m}$ is a vector subspace of $\mathfrak{g}$.

The Lie algebra $\mathfrak{g}$ admits a basis $\left\{U_{1}, U_{2}, U_{3}, U_{4}, U_{5}\right\}$, where $\left\{U_{1}, U_{2}, U_{3}\right.$, $\left.U_{4}\right\}$ is an orthogonal basis of $\mathfrak{m}$ and $\left\{U_{5}\right\}$ a basis of $\mathfrak{h}$, such that the Lie bracket $[$,$] on \mathfrak{g}$ and the scalar product $\langle$,$\rangle on \mathfrak{m}$ are given by

\begin{tabular}{c|ccccc}
{$[]$,} & $U_{1}$ & $U_{2}$ & $U_{3}$ & $U_{4}$ & $U_{5}$ \\
\hline$U_{1}$ & 0 & 0 & $-\delta U_{1}$ & $\delta U_{2}$ & $U_{2}$ \\
$U_{2}$ & 0 & 0 & $\delta U_{2}$ & $\delta U_{1}$ & $-U_{1}$ \\
$U_{3}$ & $\delta U_{1}$ & $-\delta U_{2}$ & 0 & $-2 \delta^{2} U_{5}$ & $-2 U_{4}$ \\
$U_{4}$ & $-\delta U_{2}$ & $-\delta U_{1}$ & $2 \delta^{2} U_{5}$ & 0 & $2 U_{3}$ \\
$U_{5}$ & $-U_{2}$ & $U_{1}$ & $2 U_{4}$ & $-2 U_{3}$ & 0
\end{tabular}

where $\delta>0$ is a real constant, and

\begin{tabular}{c|cccc}
$\langle\rangle$, & $U_{1}$ & $U_{2}$ & $U_{3}$ & $U_{4}$ \\
\hline$U_{1}$ & 1 & 0 & 0 & 0 \\
$U_{2}$ & 0 & 1 & 0 & 0 \\
$U_{3}$ & 0 & 0 & -2 & 0 \\
$U_{4}$ & 0 & 0 & 0 & -2
\end{tabular},

respectively.

We now recall the following result on the curvature tensor and the Ricci tensor of four-dimensional generalized symmetric spaces of type A (see [3]).

Lemma 3.1. Let $M$ be a four-dimensional generalized symmetric space of type $A$ and signature $(2,2)$. Then, there exist a pseudo-orthonormal frame field $\left\{e_{1}=U_{1}, e_{2}=U_{2}, e_{3}=\frac{1}{\sqrt{2}} U_{3}, e_{4}=\frac{1}{\sqrt{2}} U_{4}\right\}$ on $M$, with $\left\langle e_{1}, e_{1}\right\rangle=\left\langle e_{2}, e_{2}\right\rangle=$ $-\left\langle e_{3}, e_{3}\right\rangle=-\left\langle e_{4}, e_{4}\right\rangle=1$. The non-vanishing components of the Levi-Civita connection $\nabla$ of $M$ are given by

$$
\begin{array}{cccc}
\nabla_{e_{1}} e_{1}=-\frac{\delta}{\sqrt{2}} e_{3}, & \nabla_{e_{1}} e_{2}=\frac{\delta}{\sqrt{2}} e_{4}, & \nabla_{e_{1}} e_{3}=-\frac{\delta}{\sqrt{2}} e_{1}, & \nabla_{e_{1}} e_{4}=\frac{\delta}{\sqrt{2}} e_{2}, \\
\nabla_{e_{2}} e_{1}=\frac{\delta}{\sqrt{2}} e_{4}, & \nabla_{e_{2}} e_{2}=\frac{\delta}{\sqrt{2}} e_{3}, & \nabla_{e_{2}} e_{3}=\frac{\delta}{\sqrt{2}} e_{2}, & \nabla_{e_{2}} e_{4}=\frac{\delta}{\sqrt{2}} e_{1} .
\end{array}
$$

The only non-zero components of the Riemann curvature tensor $R(X, Y$, $Z, W)=g(R(X, Y) Z, W)$, with respect to $\left\{e_{1}, e_{2}, e_{3}, e_{4}\right\}$, are

$$
\begin{gathered}
R_{1212}=-R_{1234}=-\delta^{2}, \\
R_{1313}=-R_{1324}=R_{1414}=R_{1423}=R_{2323}=R_{2424}=-\frac{\delta^{2}}{2}
\end{gathered}
$$

and the non-zero components of the Ricci tensor are given by

$$
\varrho_{33}=\varrho_{44}=-\delta^{2} .
$$

Now, let $\mathrm{D} \in \operatorname{Der}(\mathfrak{g})$, where $\mathfrak{g}$ is the Lie algebra in (3.1). Put

$\mathrm{D} U_{l}=\lambda_{l}^{1} U_{1}+\lambda_{l}^{2} U_{2}+\lambda_{l}^{3} U_{3}+\lambda_{l}^{4} U_{4}+\lambda_{l}^{5} U_{5}$ for all $l=1, \ldots, 5$.

Starting from (3.1), we can write down (1.2), getting 


$$
\begin{aligned}
& \lambda_{3}^{5}+\delta\left(2 \lambda_{1}^{2}+\lambda_{3}^{4}\right)=0, \quad \lambda_{3}^{5}+\delta\left(2 \lambda_{2}^{1}-\lambda_{3}^{4}\right)=0, \\
& \lambda_{4}^{5}+\delta\left(\lambda_{1}^{1}-\lambda_{2}^{2}\right)=0, \quad \lambda_{2}^{1}-\lambda_{1}^{2}+\lambda_{4}^{3}=0, \\
& \lambda_{1}^{1}-\lambda_{2}^{2}+\delta \lambda_{5}^{4}=0, \quad \lambda_{1}^{2}+\lambda_{2}^{1}+\delta \lambda_{5}^{3}=0, \\
& \lambda_{3}^{2}+\lambda_{4}^{1}+2 \delta \lambda_{5}^{1}=0, \quad \lambda_{3}^{1}-\lambda_{4}^{2}+2 \delta \lambda_{5}^{2}=0, \\
& 2 \lambda_{4}^{1}-\lambda_{3}^{2}+\delta \lambda_{5}^{1}=0, \quad 2 \lambda_{4}^{2}+\lambda_{3}^{1}-\delta \lambda_{5}^{2}=0, \\
& \lambda_{4}^{1}-2 \lambda_{3}^{2}-\delta \lambda_{5}^{1}=0, \quad \lambda_{4}^{2}+2 \lambda_{3}^{1}+\delta \lambda_{5}^{2}=0, \\
& \lambda_{3}^{5}=\delta^{2} \lambda_{5}^{3}, \quad \lambda_{4}^{3}=-\lambda_{3}^{4}, \quad \lambda_{4}^{5}=\delta^{2} \lambda_{5}^{4}, \\
& \lambda_{1}^{3}=\lambda_{1}^{4}=\lambda_{1}^{5}=\lambda_{2}^{3}=\lambda_{2}^{4}=\lambda_{2}^{5}=\lambda_{3}^{3}=\lambda_{4}^{4}=\lambda_{5}^{5}=0 .
\end{aligned}
$$

A standard computation proves that all solutions of (3.2) are given by

$$
\begin{array}{ll}
\lambda_{2}^{1}=-\lambda_{1}^{2}-\delta \lambda_{5}^{3}, & \lambda_{2}^{2}=\lambda_{1}^{1}+\delta \lambda_{5}^{4}, \quad \lambda_{3}^{1}=-\lambda_{4}^{2}=-\delta \lambda_{5}^{2}, \\
\lambda_{3}^{2}=\lambda_{4}^{1}=-\delta \lambda_{5}^{1}, & \lambda_{4}^{3}=-\lambda_{3}^{4}=2 \lambda_{1}^{2}+\delta \lambda_{5}^{3} .
\end{array}
$$

So, we have proved the following

Lemma 3.2. Let $\mathfrak{g}=\mathfrak{h} \oplus \mathfrak{m}$ be the Lie algebra in (3.1). Then $\mathrm{D} \in \operatorname{Der}(\mathfrak{g})$ if and only if

$$
\mathrm{D}=\left(\begin{array}{ccccc}
\lambda_{1}^{1} & -\lambda_{1}^{2}-\delta \lambda_{5}^{3} & -\delta \lambda_{5}^{2} & -\delta \lambda_{5}^{1} & \lambda_{5}^{1} \\
\lambda_{1}^{2} & \lambda_{1}^{1}+\delta \lambda_{5}^{4} & -\delta \lambda_{5}^{1} & \delta \lambda_{5}^{2} & \lambda_{5}^{2} \\
0 & 0 & 0 & 2 \lambda_{1}^{2}+\delta \lambda_{5}^{3} & \lambda_{5}^{3} \\
0 & 0 & -2 \lambda_{1}^{2}-\delta \lambda_{5}^{3} & 0 & \lambda_{5}^{4} \\
0 & 0 & \delta^{2} \lambda_{5}^{3} & \delta^{2} \lambda_{5}^{4} & 0
\end{array}\right)
$$

Using the lemma above, we now prove the following.

Theorem 3.3. Any four-dimensional generalized symmetric space $(M=G / H, g)$ of type $A$ and signature $(2,2)$ is an algebraic Ricci soliton. In particular,

$$
\operatorname{pr} \circ \mathrm{D}=\left(\begin{array}{cccc}
-\delta^{2} & 0 & 0 & 0 \\
0 & -\delta^{2} & 0 & 0 \\
0 & 0 & 0 & 0 \\
0 & 0 & 0 & 0
\end{array}\right) \text { and } c=\delta^{2}
$$

Proof. Using Lemma 3.1 we obtain that the Ricci operator of ( $M=G / H, g)$ is given, with respect to the basis $\left\{U_{1}, U_{2}, U_{3}, U_{4}, U_{5}\right\}$, by

$$
\operatorname{Ric}=\left(\begin{array}{cccc}
0 & 0 & 0 & 0 \\
0 & 0 & 0 & 0 \\
0 & 0 & \delta^{2} & 0 \\
0 & 0 & 0 & \delta^{2}
\end{array}\right)
$$

Hence, the algebraic Ricci soliton condition (1.4) on $M$ is satisfied if and only if

$$
\lambda_{1}^{1}=-c=-\delta^{2}, \quad \lambda_{1}^{2}=\lambda_{5}^{1}=\lambda_{5}^{2}=\lambda_{5}^{3}=\lambda_{5}^{4}=0 .
$$


Remark 3.4. Riemannian spaces of type A which are algebraic Ricci solitons are obtained as follows. Changing $U_{3}$ and $U_{4}$ in the pseudo-Riemannian case to $(1 / \delta) U_{3}$ and $(1 / \delta) U_{4}$ and letting $\varrho=-\delta^{2}$, we obtain the Lie bracket of the Riemannian case. It is easy to check that one thus get an algebraic Ricci soliton.

\section{Spaces of Type B}

Let $(M=G / H, g)$ be a non-symmetric simply-connected four-dimensional generalized symmetric space of type $B$ and signature $(2,2)$. Then, $\mathfrak{g}=\mathfrak{h} \oplus \mathfrak{m}$ and $\left\{U_{1}, U_{2}, U_{3}, U_{4}\right\}$ and $\left\{U_{5}\right\}$ are bases of $\mathfrak{m}$ and $\mathfrak{h}$, respectively, such that the Lie bracket $[$,$] on \mathfrak{g}$ and the scalar product $\langle$,$\rangle on \mathfrak{m}$ are given by

\begin{tabular}{c|ccccc}
{$[]$,} & $U_{1}$ & $U_{2}$ & $U_{3}$ & $U_{4}$ & $U_{5}$ \\
\hline$U_{1}$ & 0 & 0 & $-U_{1}$ & $\varepsilon U_{5}+U_{2}$ & 0 \\
$U_{2}$ & 0 & 0 & $-\varepsilon U_{5}+U_{2}$ & $U_{1}$ & 0 \\
$U_{3}$ & $U_{1}$ & $\varepsilon U_{5}-U_{2}$ & 0 & 0 & $2 U_{2}$ \\
$U_{4}$ & $-\varepsilon U_{5}-U_{2}$ & $-U_{1}$ & 0 & 0 & $-2 U_{1}$ \\
$U_{5}$ & 0 & 0 & $-2 U_{2}$ & $2 U_{1}$ & 0
\end{tabular},

where $\varepsilon= \pm 1$, and

\begin{tabular}{c|cccc}
$\langle\rangle$, & $U_{1}$ & $U_{2}$ & $U_{3}$ & $U_{4}$ \\
\hline$U_{1}$ & 0 & 0 & -1 & 0 \\
$U_{2}$ & 0 & 0 & 0 & -1 \\
$U_{3}$ & -1 & 0 & $2 \lambda$ & 0 \\
$U_{4}$ & 0 & -1 & 0 & $2 \lambda$
\end{tabular}

respectively.

The following result was proven in [3].

Lemma 4.1. Let $M$ be a four-dimensional generalized symmetric space of type $B$ and signature $(2,2)$. There exists a pseudo-orthonormal frame field

$$
\begin{array}{lll}
e_{1}=\left(\lambda-\frac{1}{2}\right) U_{1}+U_{2}, & e_{2}=\left(\lambda-\frac{1}{2}\right) U_{3}+U_{4}, \\
e_{3}=\left(\lambda+\frac{1}{2}\right) U_{1}+U_{2}, & e_{4}=\left(\lambda+\frac{1}{2}\right) U_{3}+U_{4},
\end{array}
$$

on $M$, with $\left\langle e_{1}, e_{1}\right\rangle=\left\langle e_{2}, e_{2}\right\rangle=-\left\langle e_{3}, e_{3}\right\rangle=-\left\langle e_{4}, e_{4}\right\rangle=1$. The Levi-Civita connection $\nabla$ of $M$ is determined by

$$
\begin{array}{llll}
\nabla_{e_{1}} e_{1}=-e_{3}, & \nabla_{e_{2}} e_{1}=e_{4}, & \nabla_{e_{3}} e_{1}=-e_{3}, & \nabla_{e_{4}} e_{1}=e_{4}, \\
\nabla_{e_{1}} e_{2}=e_{4}, & \nabla_{e_{2}} e_{2}=e_{3}, & \nabla_{e_{3}} e_{2}=e_{4}, & \nabla_{e_{4}} e_{2}=e_{3}, \\
\nabla_{e_{1}} e_{3}=-e_{1}, & \nabla_{e_{2}} e_{3}=e_{2}, & \nabla_{e_{3}} e_{3}=-e_{1}, & \nabla_{e_{4}} e_{3}=e_{2}, \\
\nabla_{e_{1}} e_{4}=e_{2}, & \nabla_{e_{2}} e_{4}=e_{1}, & \nabla_{e_{3}} e_{4}=e_{2}, & \nabla_{e_{4}} e_{4}=e_{1} .
\end{array}
$$


The only non-zero components of the Riemann curvature tensor $R$, with respect to $\left\{e_{1}, e_{2}, e_{3}, e_{4}\right\}$, are

$$
R_{1212}=R_{1214}=-R_{1223}=-R_{1234}=-R_{1434}=R_{2334}=-R_{3434}=-2
$$

and the non-zero components of the Ricci tensor are given by

$$
\varrho_{11}=\varrho_{22}=\varrho_{33}=\varrho_{44}=-2, \quad \varrho_{13}=\varrho_{24}=-4 .
$$

Next, let $\mathrm{D} \in \operatorname{Der}(\mathfrak{g})$, where $\mathfrak{g}$ is the Lie algebra in (4.1) and put

$\mathrm{D} U_{l}=\lambda_{l}^{1} U_{1}+\lambda_{l}^{2} U_{2}+\lambda_{l}^{3} U_{3}+\lambda_{l}^{4} U_{4}+\lambda_{l}^{5} U_{5}$ for all $l=1, \ldots, 5$.

Using (4.1), we prove that (1.2) is satisfied if and only if

$$
\begin{aligned}
& \lambda_{3}^{4}+2\left(\lambda_{1}^{2}-\lambda_{1}^{5}\right)=0, \quad \lambda_{1}^{5}+\varepsilon\left(\lambda_{3}^{4}-\lambda_{1}^{2}\right)=0, \\
& \lambda_{1}^{2}-\lambda_{4}^{3}+2 \lambda_{1}^{5}-\varepsilon \lambda_{5}^{1}-\lambda_{2}^{1}=0, \quad \lambda_{1}^{1}+\lambda_{4}^{4}-\varepsilon \lambda_{5}^{2}-\lambda_{2}^{2}=0, \\
& \lambda_{2}^{5}-\varepsilon\left(\lambda_{1}^{1}+\lambda_{4}^{4}-\lambda_{5}^{5}\right)=0, \quad \lambda_{3}^{4}-2 \lambda_{2}^{1}+\varepsilon \lambda_{5}^{1}=0, \\
& \lambda_{2}^{5}+\varepsilon\left(\lambda_{2}^{2}-\lambda_{5}^{5}\right)=0, \quad \lambda_{1}^{1}-\lambda_{2}^{2}-\lambda_{4}^{4}-2 \lambda_{2}^{5}=0, \\
& \lambda_{1}^{2}-\lambda_{2}^{1}-\lambda_{4}^{3}=0, \lambda_{1}^{5}+\varepsilon\left(\lambda_{4}^{3}-\lambda_{2}^{1}\right)=0 \\
& \lambda_{3}^{2}+\lambda_{4}^{1}+2 \lambda_{3}^{5}=0, \lambda_{3}^{1}-\lambda_{4}^{2}+2 \lambda_{4}^{5}=0 \\
& \lambda_{5}^{1}-2\left(\lambda_{2}^{1}+\lambda_{3}^{4}\right)=0, \lambda_{5}^{2}+2\left(\lambda_{2}^{2}-\lambda_{5}^{5}\right)=0 \\
& \lambda_{5}^{2}+2\left(-\lambda_{1}^{1}+\lambda_{4}^{4}+\lambda_{5}^{5}\right)=0, \quad \lambda_{5}^{1}-2\left(\lambda_{1}^{2}+\lambda_{4}^{3}\right)=0 \\
& \lambda_{4}^{2}=-\lambda_{3}^{1}, \quad \lambda_{5}^{1}=2 \varepsilon \lambda_{1}^{5}, \quad \lambda_{5}^{2}=2 \varepsilon \lambda_{2}^{5}, \\
& \lambda_{1}^{3}=\lambda_{1}^{4}=\lambda_{2}^{3}=\lambda_{2}^{4}=\lambda_{3}^{3}=\lambda_{5}^{3}=\lambda_{5}^{4}=0 .
\end{aligned}
$$

So, we need to consider two cases:

- If $\varepsilon=1$, it is easily seen that all solutions of (4.2) are given by

$$
\begin{aligned}
\lambda_{1}^{5}=\lambda_{2}^{1} & =\lambda_{1}^{2}, \quad \lambda_{4}^{1}=-\lambda_{3}^{2}-2 \lambda_{3}^{5}, \quad \lambda_{4}^{2}=\lambda_{4}^{5}=-\lambda_{3}^{1}, \quad \lambda_{2}^{2}=\lambda_{1}^{1}-2 \lambda_{2}^{5}, \\
\lambda_{5}^{1} & =2 \lambda_{1}^{2}, \quad \lambda_{5}^{2}=2 \lambda_{2}^{5}, \quad \lambda_{5}^{5}=\lambda_{1}^{1}-\lambda_{2}^{5}, \quad \lambda_{3}^{4}=\lambda_{4}^{3}=\lambda_{4}^{4}=0 .
\end{aligned}
$$

- If $\varepsilon=-1$, all solutions of (4.2) are given by

$$
\begin{aligned}
& \lambda_{4}^{1}=-\lambda_{3}^{2}-2 \lambda_{3}^{5}, \quad \lambda_{4}^{2}=\lambda_{4}^{5}=-\lambda_{3}^{1}, \quad \lambda_{5}^{5}=\lambda_{2}^{2}=\lambda_{1}^{1}, \\
& \lambda_{1}^{2}=\lambda_{1}^{5}=\lambda_{2}^{1}=\lambda_{2}^{5}=\lambda_{3}^{4}=\lambda_{4}^{3}=\lambda_{4}^{4}=\lambda_{5}^{1}=\lambda_{5}^{2}=0 .
\end{aligned}
$$

Therefore, we have proved the following

Lemma 4.2. Let $\mathfrak{g}=\mathfrak{h} \oplus \mathfrak{m}$ be the Lie algebra in (4.1). Then $\mathrm{D} \in \operatorname{Der}(\mathfrak{g})$ if and only if

- $\varepsilon=1$ :

$$
\mathrm{D}=\left(\begin{array}{ccccc}
\lambda_{1}^{1} & \lambda_{1}^{2} & \lambda_{3}^{1} & -\lambda_{3}^{2}-2 \lambda_{3}^{5} & 2 \lambda_{1}^{2} \\
\lambda_{1}^{2} & \lambda_{1}^{1}-2 \lambda_{2}^{5} & \lambda_{3}^{2} & -\lambda_{3}^{1} & 2 \lambda_{2}^{5} \\
0 & 0 & 0 & 0 & 0 \\
0 & 0 & 0 & 0 & 0 \\
\lambda_{1}^{2} & \lambda_{2}^{5} & \lambda_{3}^{5} & -\lambda_{3}^{1} & \lambda_{1}^{1}-\lambda_{2}^{5}
\end{array}\right)
$$


- $\varepsilon=-1$ :

$$
\mathrm{D}=\left(\begin{array}{ccccc}
\lambda_{1}^{1} & 0 & \lambda_{3}^{1} & -\lambda_{3}^{2}-2 \lambda_{3}^{5} & 0 \\
0 & \lambda_{1}^{1} & \lambda_{3}^{2} & -\lambda_{3}^{1} & 0 \\
0 & 0 & 0 & 0 & 0 \\
0 & 0 & 0 & 0 & 0 \\
0 & \lambda_{2}^{5} & \lambda_{3}^{5} & -\lambda_{3}^{1} & \lambda_{1}^{1}
\end{array}\right)
$$

Using the lemma above, we now prove the following.

Theorem 4.3. Any 4-dimensional generalized symmetric space $(M=G / H, g)$ of type $B$ is not an algebraic Ricci soliton.

Proof. Using Lemma 4.1 we obtain that the Ricci operator of ( $M=G / H, g)$ is given, with respect to the basis $\left\{U_{1}, U_{2}, U_{3}, U_{4}, U_{5}\right\}$, by

$$
\operatorname{Ric}=\left(\begin{array}{cccc}
-4 \lambda & 0 & 4 \lambda^{2}+3 & 0 \\
0 & -4 \lambda & 0 & 4 \lambda^{2}+3 \\
-4 & 0 & 4 \lambda & 0 \\
0 & -4 & 0 & 4 \lambda
\end{array}\right)
$$

Hence it follows, from the lemma above, that the algebraic Ricci soliton condition (1.4) on $M$ is not satisfied.

\section{Spaces of Type C}

Let $(M=G, g)$ be a non-symmetric simply-connected four-dimensional symmetric space of type $\mathrm{C}$. Without loss of generality, we assume that the signature is $(3,1)$. The Lie algebra $\mathfrak{g}$ admits a basis $\left\{U_{1}, U_{2}, U_{3}, U_{4}\right\}$, such that the Lie bracket $[$,$] and the scalar product \langle$,$\rangle on \mathfrak{g}$ are given by

\begin{tabular}{c|cccc}
{$[]$,} & $U_{1}$ & $U_{2}$ & $U_{3}$ & $U_{4}$ \\
\hline$U_{1}$ & 0 & 0 & 0 & $-U_{1}$ \\
$U_{2}$ & 0 & 0 & 0 & $U_{2}$ \\
$U_{3}$ & 0 & 0 & 0 & 0 \\
$U_{4}$ & $U_{1}$ & $-U_{2}$ & 0 & 0
\end{tabular}

and

\begin{tabular}{c|cccc}
$\langle\rangle$, & $U_{1}$ & $U_{2}$ & $U_{3}$ & $U_{4}$ \\
\hline$U_{1}$ & 1 & 0 & 0 & 0 \\
$U_{2}$ & 0 & 1 & 0 & 0 \\
$U_{3}$ & 0 & 0 & 0 & $1 / 2$ \\
$U_{4}$ & 0 & 0 & $1 / 2$ & 0
\end{tabular},

respectively.

The following result was proven in [6]. 
Lemma 5.1. Let $M$ be a four-dimensional symmetric space of type $C$ and signature $(3,1)$. There exists a pseudo-orthonormal frame field

$$
e_{1}=U_{1}, \quad e_{2}=U_{2}, \quad e_{3}=U_{3}+U_{4}, \quad e_{4}=U_{3}-U_{4},
$$

on $M$, with $\left\langle e_{1}, e_{1}\right\rangle=\left\langle e_{2}, e_{2}\right\rangle=\left\langle e_{3}, e_{3}\right\rangle=-\left\langle e_{4}, e_{4}\right\rangle=1$. The non-vanishing components of the Levi-Civita connection $\nabla$ of $M$ are given by

$\nabla_{e_{1}} e_{1}=-\nabla_{e_{2}} e_{2}=e_{3}+e_{4}, \quad \nabla_{e_{1}} e_{4}=-\nabla_{e_{1}} e_{3}=e_{1}, \quad \nabla_{e_{2}} e_{3}=-\nabla_{e_{2}} e_{4}=e_{2}$.

The non-zero components of the Riemann curvature tensor $R$, with respect to $\left\{e_{1}, e_{2}, e_{3}, e_{4}\right\}$, are

$$
R_{1313}=-R_{1314}=R_{1414}=R_{2323}=-R_{2324}=R_{2424}=-1
$$

and the non-zero components of the Ricci tensor are given by

$$
\varrho_{33}=\varrho_{22}=-\varrho_{34}=-2 .
$$

Next, put $\mathrm{D} U_{l}=\lambda_{l}^{1} U_{1}+\lambda_{l}^{2} U_{2}+\lambda_{l}^{3} U_{3}+\lambda_{l}^{4} U_{4}$ for all $l=1, \ldots, 4$, where $\left\{U_{1}, U_{2}, U_{3}, U_{4}\right\}$ is the basis in (5.1). Standard computations prove that $\mathrm{D} \in$ $\operatorname{Der}(\mathfrak{g})$ if and only if

$$
\lambda_{1}^{2}=\lambda_{1}^{3}=\lambda_{1}^{4}=\lambda_{2}^{1}=\lambda_{2}^{3}=\lambda_{2}^{4}=\lambda_{3}^{1}=\lambda_{3}^{2}=\lambda_{3}^{4}=\lambda_{4}^{4}=0 .
$$

So, we deduce the following

Lemma 5.2. Let $\mathfrak{g}=\mathfrak{h} \oplus \mathfrak{m}$ be the Lie algebra in (5.1). Then $\mathrm{D} \in \operatorname{Der}(\mathfrak{g})$ if and only if

$$
\mathrm{D}=\left(\begin{array}{cccc}
\lambda_{1}^{1} & 0 & 0 & \lambda_{4}^{1} \\
0 & \lambda_{2}^{2} & 0 & \lambda_{4}^{2} \\
0 & 0 & \lambda_{3}^{3} & \lambda_{4}^{3} \\
0 & 0 & 0 & 0
\end{array}\right)
$$

We can now prove the following.

Theorem 5.3. Any non-symmetric simply-connected four-dimensional symmetric space $(M=G / H, g)$ of type $C$ is an algebraic Ricci soliton. In particular,

$$
\mathrm{D}=\operatorname{Ric}=\left(\begin{array}{cccc}
0 & 0 & 0 & 0 \\
0 & 0 & 0 & 0 \\
0 & 0 & 0 & -4 \\
0 & 0 & 0 & 0
\end{array}\right) \text { and } c=0
$$

Proof. Using Lemma 5.1 we write down the Ricci operator of $(M=G / H, g)$, with respect to the basis $\left\{U_{1}, U_{2}, U_{3}, U_{4}\right\}$, getting

$$
\text { Ric }=\left(\begin{array}{cccc}
0 & 0 & 0 & 0 \\
0 & 0 & 0 & 0 \\
0 & 0 & 0 & -4 \\
0 & 0 & 0 & 0
\end{array}\right)
$$


Thus, using the lemma above, we obtain that the algebraic Ricci soliton condition (1.4) on $M$ is satisfied if and only if

$$
\lambda_{1}^{1}=\lambda_{2}^{2}=\lambda_{3}^{3}=\lambda_{4}^{1}=\lambda_{4}^{2}=c=0 \quad \text { and } \quad \lambda_{4}^{3}=-4 .
$$

\section{Spaces of Type D}

Let $(M=G / H, g)$ be a four-dimensional generalized symmetric space of type $\mathrm{D}$ and signature $(2,2)$. The Lie algebra $\mathfrak{g}=\mathfrak{h} \oplus \mathfrak{m}$ of the Lie group $G$ admits a basis $\left\{U_{1}, U_{2}, U_{3}, U_{4}, U_{5}\right\}$, where $\left\{U_{1}, U_{2}, U_{3}, U_{4}\right\}$ and $\left\{U_{5}\right\}$ are bases of $\mathfrak{m}$ and of $\mathfrak{h}$, respectively, such that

\begin{tabular}{c|ccccc}
{$[]$,} & $U_{1}$ & $U_{2}$ & $U_{3}$ & $U_{4}$ & $U_{5}$ \\
\hline$U_{1}$ & 0 & 0 & 0 & $-U_{2}$ & $U_{1}$ \\
$U_{2}$ & 0 & 0 & $-U_{1}$ & 0 & $-U_{2}$ \\
$U_{3}$ & 0 & $U_{1}$ & 0 & $-U_{5}$ & $2 U_{3}$ \\
$U_{4}$ & $U_{2}$ & 0 & $U_{5}$ & 0 & $-2 U_{4}$ \\
$U_{5}$ & $-U_{1}$ & $U_{2}$ & $-2 U_{3}$ & $2 U_{4}$ & 0
\end{tabular}

and

\begin{tabular}{c|cccc}
$\langle\rangle$, & $U_{1}$ & $U_{2}$ & $U_{3}$ & $U_{4}$ \\
\hline$U_{1}$ & 0 & 1 & 0 & 0 \\
$U_{2}$ & 1 & 0 & 0 & 0 \\
$U_{3}$ & 0 & 0 & 0 & $\lambda$ \\
$U_{4}$ & 0 & 0 & $\lambda$ & 0
\end{tabular}

where $\lambda \neq 0$ is a real constant.

The following result was proven in [3].

Lemma 6.1. Let $M$ be a four-dimensional generalized symmetric space of type $D$ and signature $(2,2)$. There exists a pseudo-orthonormal frame field

$$
\begin{gathered}
e_{1}=\frac{1}{\sqrt{2}}\left(U_{1}+U_{2}\right), \quad e_{2}=\frac{1}{\sqrt{2|\lambda|}} t\left(U_{3}+\varepsilon U_{4}\right), \\
e_{3}=\frac{1}{\sqrt{2}}\left(U_{1}-U_{2}\right), \quad e_{4}=\frac{1}{\sqrt{2|\lambda|}}\left(U_{3}-\varepsilon U_{4}\right),
\end{gathered}
$$

on $M$, with $\varepsilon= \pm 1$ and $\left\langle e_{1}, e_{1}\right\rangle=\left\langle e_{2}, e_{2}\right\rangle=-\left\langle e_{3}, e_{3}\right\rangle=-\left\langle e_{4}, e_{4}\right\rangle=1$. The non-vanishing components of the Levi-Civita connection $\nabla$ of $M$ are given by

$$
\begin{aligned}
& \nabla_{e_{1}} e_{1}=\frac{1}{2 \sqrt{2|\lambda|}}\left((\varepsilon+1) e_{2}+(\varepsilon-1) e_{4}\right), \\
& \nabla_{e_{1}} e_{2}=-\frac{1}{2 \sqrt{2|\lambda|}}\left((\varepsilon+1) e_{1}-(\varepsilon-1) e_{3}\right), \\
& \nabla_{e_{1}} e_{3}=\frac{1}{2 \sqrt{2|\lambda|}}\left((\varepsilon-1) e_{2}+(\varepsilon+1) e_{4}\right),
\end{aligned}
$$




$$
\begin{aligned}
& \nabla_{e_{1}} e_{4}=\frac{1}{2 \sqrt{2|\lambda|}}\left((\varepsilon-1) e_{1}-(\varepsilon+1) e_{3}\right), \\
& \nabla_{e_{3}} e_{1}=\frac{1}{2 \sqrt{2|\lambda|}}\left((\varepsilon-1) e_{2}+(\varepsilon+1) e_{4}\right), \\
& \nabla_{e_{3}} e_{2}=\frac{1}{2 \sqrt{2|\lambda|}}\left((1-\varepsilon) e_{1}+(\varepsilon+1) e_{3}\right), \\
& \nabla_{e_{3}} e_{3}=\frac{1}{2 \sqrt{2|\lambda|}}\left((\varepsilon+1) e_{2}+(\varepsilon-1) e_{4}\right), \\
& \nabla_{e_{3}} e_{4}=\frac{1}{2 \sqrt{2|\lambda|}}\left((\varepsilon+1) e_{1}-(\varepsilon-1) e_{3}\right) .
\end{aligned}
$$

The non-zero components of the Riemann curvature tensor, with respect to $\left\{e_{1}, e_{2}, e_{3}, e_{4}\right\}$, are

$$
\begin{gathered}
R_{1212}=-R_{1234}=-R_{1414}=-R_{1423}=-R_{2323}=R_{3434}=-\frac{1}{2 \lambda}, \\
R_{1313}=-R_{1324}=-\frac{1}{\lambda}
\end{gathered}
$$

and the non-zero components of the Ricci tensor are given by

$$
\varrho\left(e_{2}, e_{2}\right)=-\varrho\left(e_{4}, e_{4}\right)=-\frac{1}{\lambda} .
$$

Now, let $\mathrm{D} \in \operatorname{Der}(\mathfrak{g})$ where $\mathfrak{g}$ is the Lie algebra in (6.1). Put

$\mathrm{D} U_{l}=\lambda_{l}^{1} U_{1}+\lambda_{l}^{2} U_{2}+\lambda_{l}^{3} U_{3}+\lambda_{l}^{4} U_{4}+\lambda_{l}^{5} U_{5}$ for all $l=1, \ldots, 5$.

Starting from (6.1), we can write down (1.2), obtaining

$$
\begin{aligned}
& \lambda_{3}^{3}=\lambda_{1}^{1}-\lambda_{2}^{2}, \lambda_{3}^{5}=\lambda_{1}^{2}, \lambda_{4}^{4}=-\lambda_{3}^{3}, \lambda_{4}^{5}=-\lambda_{2}^{1}, \\
& \lambda_{5}^{1}=-\lambda_{4}^{2}, \lambda_{5}^{2}=\lambda_{3}^{1}, \lambda_{5}^{3}=2 \lambda_{2}^{1}, \lambda_{5}^{4}=-2 \lambda_{1}^{2}, \\
& \lambda_{1}^{3}=\lambda_{1}^{4}=\lambda_{1}^{5}=\lambda_{2}^{3}=\lambda_{2}^{4}=\lambda_{2}^{5}=\lambda_{3}^{2}=\lambda_{3}^{4}=\lambda_{4}^{1}=\lambda_{4}^{3}=\lambda_{5}^{5}=0 .
\end{aligned}
$$

We deduce the following.

Lemma 6.2. Let $\mathfrak{g}=\mathfrak{h} \oplus \mathfrak{m}$ be the Lie algebra in (6.1). Then $\mathrm{D} \in \operatorname{Der}(\mathfrak{g})$ if and only if

$$
\mathrm{D}=\left(\begin{array}{ccccc}
\lambda_{1}^{1} & \lambda_{2}^{1} & \lambda_{3}^{1} & 0 & -\lambda_{4}^{2} \\
\lambda_{1}^{2} & \lambda_{2}^{2} & 0 & \lambda_{4}^{2} & \lambda_{3}^{1} \\
0 & 0 & \lambda_{1}^{1}-\lambda_{2}^{2} & 0 & 2 \lambda_{2}^{1} \\
0 & 0 & 0 & \lambda_{2}^{2}-\lambda_{1}^{1} & -2 \lambda_{1}^{2} \\
0 & 0 & \lambda_{1}^{2} & -\lambda_{2}^{1} & 0
\end{array}\right)
$$

Using the lemma above, we prove the following. 
Theorem 6.3. Any four-dimensional generalized symmetric space $(M=G / H, g)$ of type $D$ is an algebraic Ricci soliton. In particular,

$$
\operatorname{pr} \circ \mathrm{D}=\left(\begin{array}{cccc}
\frac{1}{\lambda} & 0 & 0 & 0 \\
0 & \frac{1}{\lambda} & 0 & 0 \\
0 & 0 & 0 & 0 \\
0 & 0 & 0 & 0
\end{array}\right) \quad \text { and } \quad \mathrm{c}=-\frac{1}{\lambda}
$$

Proof. Using Lemma 6.1 we write down the Ricci operator of $(M=G / H, g)$, with respect to the basis $\left\{U_{1}, U_{2}, U_{3}, U_{4}\right\}$, getting

$$
\operatorname{Ric}=\left(\begin{array}{cccc}
0 & 0 & 0 & 0 \\
0 & 0 & 0 & 0 \\
0 & 0 & -\frac{1}{\lambda} & 0 \\
0 & 0 & 0 & -\frac{1}{\lambda}
\end{array}\right)
$$

Using the lemma above, we obtain that the algebraic Ricci soliton condition (1.4) on $M$ is satisfied if and only if

$$
\lambda_{1}^{1}=\lambda_{2}^{2}=-c=\frac{1}{\lambda} \quad \text { and } \quad \lambda_{1}^{2}=\lambda_{2}^{1}=\lambda_{3}^{1}=\lambda_{3}^{3}=\lambda_{4}^{2}=0 .
$$

\section{Acknowledgements}

The authors wish to express their gratitude to the referee and to Prof. P.M. Gadea for their valuable remarks that have improved the paper. The authors would like to thank Prof. E. Garcia-Rio, Prof. H. Tamaru and Prof. J. Inoguchi for their comments.

Open Access. This article is distributed under the terms of the Creative Commons Attribution License which permits any use, distribution, and reproduction in any medium, provided the original author(s) and the source are credited.

\section{References}

[1] Batat, W., Onda, K.: Algebraic Ricci solitons on three-dimensional Lorentzian lie groups (arXiv:1112.2455)

[2] Calvaruso, G.: Homogeneous structures on three-dimensional Lorentzian manifolds. J. Geom. Phys. 57(4), 1279-1291 (2007)

[3] Calvaruso, G., de Leo, B.: Curvature properties of four-dimensional generalized symmetric spaces. J. Geom. 90, 30-46 (2008)

[4] Cerny, J., Kowalski, O.: Classification of generalized symmetric pseudo-Riemannian spaces of dimension $n=4$. Tensor N.S. 38, 256-267 (1982)

[5] de Leo, B., Marinosci, R.A.: Homogeneous geodesics of four-dimensional generalized symmetric pseudo-Riemannian spaces. Publ.Math. Debrecen. 73, 341360 (2008) 
[6] de Leo, B., der Veken, J.V.: Totally geodesic hypersurfaces of four-dimensional generalized symmetric spaces. Geometriae Dedicata 159(1), 373-387 (2012)

[7] Fels, M.E., Renner, A.G.: Non-reductive homogeneous pseudo-Riemannian manifolds of dimension four. Can. J. Math. 58(2), 282-311 (2006)

[8] Jablonski, M.: Homogeneous Ricci solitons (arXiv:1109.6556)

[9] Lauret, J.: Ricci soliton homogeneous nilmanifolds. Math. Ann. 319(4), 715-733 (2001)

[10] Onda, K.: Examples of algebraic Ricci solitons in the pseudo-Riemannian case (arXiv: 1112.0424)

[11] O'Neill, B.: Semi-Riemannian geometry. Academic Press, New York (1983)

W. Batat

École Nationale Polytechnique d'Oran

BP 1523 El M'naouar

31000 Oran

Algeria

e-mail: batatwafa@yahoo.fr

K. Onda

Graduate School of Mathematics

Nagoya University Furocho, Chikusaku

464-860 Nagoya

Japan

e-mail: kensuke.onda@math.nagoya-u.ac.jp

Received: December 28, 2011.

Accepted: April 13, 2013. 